\title{
Downlink Scheduling with Transmission Strategy Selection for Two-cell MIMO Networks
}

\author{
Binglai Niu, Vincent W.S. Wong, and Robert Schober \\ Department of Electrical and Computer Engineering \\ The University of British Columbia, Vancouver, British Columbia, Canada \\ E-mail: \{bniu, vincentw, rschober\}@ece.ubc.ca
}

\begin{abstract}
Multiple-input multiple-output (MIMO) processing is a promising technique to achieve high speed data service in next generation cellular networks. However, the performance of multi-cell MIMO networks is limited by the cell-edge users who experience significant inter-cell interference. In this paper, we propose to use interference alignment to mitigate interference experienced by these cell-edge users, and spatial multiplexing for users with less interference. To achieve the optimal performance in a two-cell MIMO network, we formulate a joint scheduling problem to select a pair of users and the corresponding transmission strategies for both cells. We design an efficient dynamic scheduling scheme based on a stochastic network optimization framework. Numerical results show that the proposed scheme achieves a superior performance compared to scheduling schemes with a single transmission strategy.
\end{abstract}

\section{INTRODUCTION}

Multiple-input multiple-output (MIMO) antenna processing has been extensively studied as a promising solution to support high speed data services in the next generation cellular systems such as Long Term Evolution (LTE) Advanced [1]. With MIMO processing, high data rates are achievable through spatial multiplexing by allowing multiple data streams to be transmitted simultaneously [2]. The potential benefit of the MIMO technology is well understood for single-cell point-topoint communication. However, in multi-cell networks, where the adjacent cells share the same frequency spectrum, the performance of spatial multiplexing is limited in the presence of interference [3]. Hence, serving the cell-edge users who experience significant inter-cell interference may become the bottleneck of the overall network performance.

In recent years, interference alignment has emerged as an effective technique to mitigate interference and to increase the capacity in interference-limited MIMO networks [4]. The main idea is that transmitters can jointly design precoding and decoding matrices based on the channel state information (CSI) such that the transmitted signals are aligned onto the same dimension (or multi-dimensional subspace) at the nonintended receiver while being distinct at the desired receiver. Through cooperation among the transmitters, interferencefree concurrent transmissions are possible and the system throughput can be improved. In the literature, several interference alignment strategies have been proposed for multicell cellular networks [5], [6], and progress has been made in designing efficient interference alignment algorithms [7]. Although interference alignment can achieve interference free transmission, an additional signal dimension is needed to accommodate the interference, and the desired signal power that lies in the interference dimension is lost. Therefore, for those users who do not experience significant interference, spatial multiplexing may outperform interference alignment. For users located at the edge of adjacent cells, where interference dominates the performance, the interference alignment strategy is desirable. In a multi-cell network where users suffer from different levels of inter-cell interference, an efficient downlink scheduling scheme is desirable which can select the users and the corresponding transmission strategies to achieve the optimal network performance. This motivates the research in this paper.

A downlink scheduling scheme aims to select in each time slot a set of users to be served with the objective to maximize a utility function. Typically, the utility function is a concave entrywise non-decreasing function of the users' long term average rate, which reflects certain fairness criteria [8]. Although several fair scheduling schemes for MIMO networks have been studied in the literature [9], [10], most of them are designed for systems with a fixed physical layer transmission strategy. A dynamic scheduling framework based on stochastic network optimization is proposed and investigated in [11], which considers two transmission strategies and non-perfect CSI. However, in [11], a single-cell model is adopted and inter-cell interference is not considered.

In this paper, we study downlink scheduling for two-cell MIMO networks. We consider the scenario where two adjacent cells share the same frequency band and users experience different levels of inter-cell interference. Different from existing works, we consider scheduling with transmission strategy selection, i.e., we propose to use interference alignment for users who experience significant inter-cell interference and spatial multiplexing for others with less interference. We formulate a joint scheduling problem, where a central scheduler selects the users as well as the corresponding transmission strategies for both cells to optimize a general utility function. Based on a stochastic network optimization framework [12], we propose an efficient dynamic scheduling scheme and evaluate its performance under the proportional fairness criterion [8]. Numerical results show that the proposed scheme achieves a superior performance compared to scheduling schemes with a single transmission strategy.

This paper is organized as follows. In Section II, we 


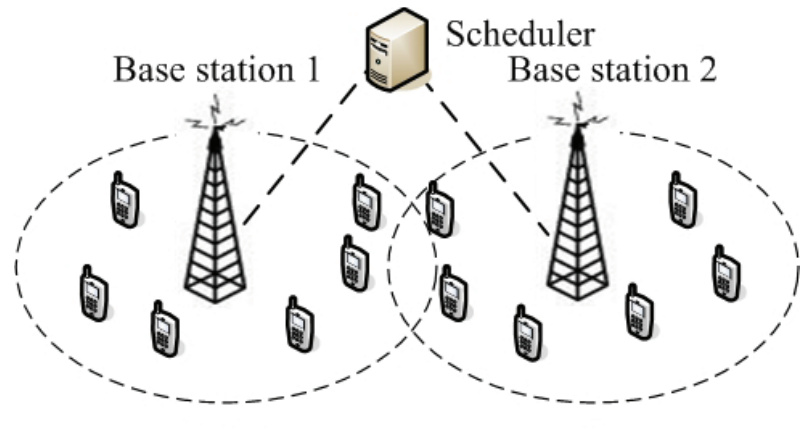

Cell 1
Cell 2

Fig. 1. A two-cell MIMO system with a central scheduler.

describe the system model and formulate the joint scheduling problem. An efficient dynamic scheduling scheme is developed in Section III. Numerical results are presented in Section IV, and conclusions are drawn in Section V.

\section{SySTEM MODEL}

We consider the downlink of a two-cell MIMO system as shown in Fig. 1 . Let $\mathcal{I}=\{1,2\}$ denote the set of cells. In cell $i \in \mathcal{I}$, a base station equipped with $N_{T}$ transmit antennas serves $K_{i}$ user terminals each of which is equipped with $N_{R}$ receive antennas. A central scheduler is deployed which connects to both base stations and schedules transmissions for the whole network. We consider a slotted system, where time is divided into slots (of equal length), and each base station serves one user in each time slot. The wireless channel is assumed to be frequency flat, where the channel gain remains constant during a time slot and is independent and identically distributed (i.i.d.) in different time slots. We denote the set of users in cell $i$ as $\mathcal{K}_{i}=\left\{l_{1}^{i}, \ldots, l_{K_{i}}^{i}\right\}$. The signal received at user $k \in \mathcal{K}_{i}$ can be represented as

$$
y_{i k}=\sqrt{g_{i k}} \mathbf{H}_{i k} x_{i}+\sqrt{g_{j k}} \mathbf{H}_{j k} x_{j}+n_{i k}, \quad i, j \in \mathcal{I}, i \neq j,
$$

where $x_{i}$ is the signal transmitted at the $i$ th base station, $\mathbf{H}_{i k} \in \mathbb{C}^{N_{R} \times N_{T}}$ is the channel matrix from the $i$ th base station to user $k$, whose elements are i.i.d. with zero mean and unit variance complex Gaussian distribution $(\mathcal{C N}(0,1))$, $g_{i k}$ is the (distance-dependent) average path gain from the $i$ th base station to user $k$, and $n_{i k}$ is additive white Gaussian noise (AWGN) with distribution $\mathcal{C N}(0,1)$. The first term on the right hand side in (1) represents the desired signal and the second term corresponds to the inter-cell interference.

We assume the channel matrices of the desired channel and the interference channel are known perfectly at the user terminals and are available to the base stations through feedback at the beginning of each time slot. This information can be passed to the scheduler to make scheduling decisions, and we assume there is no information loss during this process. We consider an infinite backlog situation where the base stations always have data to transmit for each user. We assume that the base stations can adapt the transmission rate based on the scheduling decisions.

\section{A. MIMO Transmission Strategies}

To achieve high data rates in MIMO networks, we consider two physical layer transmission strategies, namely, spatial multiplexing and interference alignment, which are described below.

i) Spatial multiplexing: As shown in [2], for a MIMO link with no interference, the transmitter can deliver multiple data streams to the receiver using spatial multiplexing. Specifically, for a $N_{T} \times N_{R}$ MIMO link, $N=\min \left\{N_{T}, N_{R}\right\}$ data streams are multiplexed by using a precoding matrix $\mathbf{V}_{i k}$ at the transmitter and are reconstructed with a decoding matrix $\mathbf{U}_{i k}$ at the receiver, where $\mathbf{V}_{i k}$ and $\mathbf{U}_{i k}$ are obtained from the singular value decomposition (SVD) of the channel matrix $\mathbf{H}_{i k}$ :

$$
\mathbf{H}_{i k}=\mathbf{U}_{i k} \boldsymbol{\Lambda}_{i k} \mathbf{V}_{i k}^{\mathrm{H}},
$$

where $\mathbf{U}_{i k} \in \mathbb{C}^{N_{R} \times N_{R}}$ and $\mathbf{V}_{i k} \in \mathbb{C}^{N_{T} \times N_{T}}$ are unitary matrices, and $\boldsymbol{\Lambda}_{i k} \in \mathbb{R}^{N_{R} \times N_{T}}$ is a rectangular matrix with non-negative diagonal elements, $\left\{\lambda_{i k, 1}, \ldots, \lambda_{i k, N}\right\}$, and all non-diagonal elements equal to zero. By applying the above precoding and decoding matrices, the MIMO link is transformed into several parallel Gaussian channels, which can support multiple data streams. The total power $P$ is distributed among the data streams using waterfilling to maximize the achievable rate (assuming there is no interference). The power allocated to the $m$ th data stream for user $k$ in cell $i$ is

$$
P_{i k, m}=\max \left\{\mu-\frac{1}{\lambda_{i k, m}^{2}}, 0\right\},
$$

and $\mu$ is chosen to satisfy $\sum_{m=1}^{N} P_{i k, m}=P$.

Similar to [5], the achievable data rate using spatial multiplexing in the presence of inter-cell interference can be derived for the two-cell MIMO network. In particular, the data rate for user $k$ in cell $i$ is

$$
\begin{aligned}
R_{i k}^{\mathrm{SM}}= & \log _{2} \operatorname{det}\left(\mathbf{I}_{N_{R}}+\left(g_{i k} \mathbf{H}_{i k} \boldsymbol{\Phi}_{S M, i} \mathbf{H}_{i k}^{\mathrm{H}}\right)\right. \\
& \left.\times\left(\mathbf{I}_{N_{R}}+g_{j k} \mathbf{H}_{j k} \boldsymbol{\Phi}_{S M, j} \mathbf{H}_{j k}^{\mathrm{H}}\right)^{-1}\right),
\end{aligned}
$$

where $\boldsymbol{\Phi}_{S M, i}=\mathbf{V}_{i k} \operatorname{diag}\left\{P_{i k, 1}, \ldots, P_{i k, N}\right\} \mathbf{V}_{i k}^{\mathrm{H}}$ is the covariance matrix of the transmitted signal at the $i$ th base station.

ii) Interference alignment: Interference alignment is used to improve the data rate for users who experience significant inter-cell interference. It allows interference-free concurrent transmissions by sacrificing some degrees of freedom. Specifically, it requires cooperation of both base stations to jointly design the transmit and receive matrices such that the interference and the desired signal lie in orthogonal subspaces at the receiver. In the two-cell MIMO network with strong interference, the $i$ th base station can transmit $d_{i}\left(d_{i} \leq N-1\right)$ data streams to user $k$ by designing precoding matrix $\widetilde{\mathbf{V}}_{i k}$ and decoding matrix $\widetilde{\mathbf{U}}_{i k}$ such that

$$
\widetilde{\mathbf{U}}_{i k}^{\mathrm{H}} \mathbf{H}_{j k} \widetilde{\mathbf{V}}_{j k}=\mathbf{0}_{d_{i}}, \quad i, j \in \mathcal{I}, i \neq j,
$$

and

$$
\operatorname{rank}\left(\widetilde{\mathbf{U}}_{i k}^{\mathrm{H}} \mathbf{H}_{i k} \widetilde{\mathbf{V}}_{i k}\right)=d_{i},
$$


where $\widetilde{\mathbf{U}}_{i k} \in \mathbb{C}^{N_{R} \times d_{i}}, \widetilde{\mathbf{V}}_{i k} \in \mathbb{C}^{N_{T} \times d_{i}}$ are truncated unitary matrices, and $\mathbf{0}_{d_{i}}$ is the $d_{i} \times 1$ all-zero vector. It can be seen that if the solution to (5) and (6) exists, then interference can be suppressed at the desired receiver. By employing the interference alignment strategy, the achievable rate for user $k$ in cell $i$ is

$$
R_{i k}^{\mathrm{IA}}=\log _{2} \operatorname{det}\left(\mathbf{I}_{d_{i}}+g_{i k} \hat{\mathbf{H}}_{i k} \boldsymbol{\Phi}_{I A, i} \hat{\mathbf{H}}_{i k}^{\mathrm{H}}\right)
$$

where $\hat{\mathrm{H}}_{i k}=\widetilde{\mathbf{U}}_{i k}^{\mathrm{H}} \mathrm{H}_{i k} \widetilde{\mathbf{V}}_{i k}$, and $\Phi_{I A, i}=\left(P / d_{i}\right) \cdot \mathbf{I}_{d_{i}}$ is the covariance matrix of the data symbols to be transmitted (without precoding) at the $i$ th base station (with equal power allocation for all data streams). For the considered two-cell system, an explicit interference alignment strategy is available [7]. In this paper, we use fixed precoding matrices at each base station and design decoding matrices for each selected user according to (5) and (6). As an example, for $N_{T}=$ $N_{R}=2$, since one dimension is needed to accommodate the interference, only one data stream can be supported. That is, $d_{i}=1$, which implies one data stream should be transmitted using both antennas. We use a fixed $2 \times 1$ unitary transmit vector $\mathbf{v}_{i k}=\mathbf{v}=\left[\begin{array}{ll}v_{1} & v_{2}\end{array}\right]^{\mathrm{T}}(\forall i \in \mathcal{I})$, where $\|\mathbf{v}\|=1$ (i.e., $\left.\left|v_{1}\right|=\left|v_{2}\right|=\sqrt{0.5}\right)$, and find the unitary decoding vector $\mathbf{u}_{i k}=\left[\begin{array}{ll}u_{i k, 1} & u_{i k, 2}\end{array}\right]^{\mathrm{T}}$ that satisfies

$$
\mathbf{u}_{i k}^{\mathrm{H}} \mathbf{H}_{j k} \mathbf{v}_{j k}=0
$$

and

$$
\left\|\mathbf{u}_{i k}\right\|^{2}=\left|u_{i k, 1}\right|^{2}+\left|u_{i k, 2}\right|^{2}=1,
$$

which is clearly feasible for a given $\mathbf{H}_{j k}$.

Note that although interference alignment can suppress the inter-cell interference, the signal power that lies in the interference subspace is lost. Therefore, when the interference is not significant, spatial multiplexing may achieve a better performance. For a system with users who experience different levels of inter-cell interference, a proper transmission strategy is desirable for each user when scheduling the transmission.

\section{B. Problem Formulation}

In order to achieve high data rates in the presence of intercell interference, cooperation of both base stations is required to implement the interference alignment strategy. In our system model, the scheduler can coordinate the base stations and jointly optimize the transmission strategy and user selection based on the CSI obtained from the base stations.

We denote the transmission rate of the $i$ th base station $\left(i \in \mathcal{I}\right.$ ) for user $k \in \mathcal{K}_{i}$ in time slot $t$ as $R_{i k}[t]$, the corresponding time average of the service rate as $\bar{R}_{i k}=$ $\lim _{t \rightarrow \infty} \frac{1}{t} \sum_{\tau=0}^{t-1} R_{i k}[\tau]$, and $\bar{R}_{i}=\left(\bar{R}_{i l_{1}^{i}}, \ldots, \bar{R}_{i l_{K_{i}}^{i}}\right)$. A fair scheduling scheme aims to maximize some concave entrywise non-decreasing utility function $\varphi(\cdot)$ of the time average rates $\bar{R}_{1}$ and $\bar{R}_{2}$ by selecting a pair of users (one from each cell) and corresponding transmission strategies in each time slot. Note that since interference alignment requires cooperation of both base stations, the same transmission strategy should be selected for them in a given time slot. Let $s[t]$ represent the transmission strategy that is selected in time slot $t$, where

$$
s[t]= \begin{cases}1, & \text { if spatial multiplexing is used, } \\ 0, & \text { if interference alignment is used, }\end{cases}
$$

and $\mathcal{S}=\{0,1\}$. Then, the optimal scheduling problem can be formulated as

$$
\begin{array}{cll}
\underset{k_{1}[t], k_{2}[t], s[t]}{\operatorname{maximize}} & \varphi\left(\bar{R}_{1}, \bar{R}_{2}\right) & \\
\text { subject to } & k_{1}[t] \in \mathcal{K}_{1}, k_{2}[t] \in \mathcal{K}_{2}, & \forall t \\
& s[t] \in \mathcal{S}, & \forall t .
\end{array}
$$

We denote $\mathcal{R}$ as the collection (feasible region) of achievable average rates $\overline{\mathbf{R}}=\left(\bar{R}_{1}, \bar{R}_{2}\right)$ under all possible scheduling schemes for our system. Suppose the optimal rates $\overline{\mathbf{R}}^{*} \in \mathcal{R}$ that maximize $\varphi\left(\bar{R}_{1}, \bar{R}_{2}\right)$ are found. From (11), the optimal scheduling scheme is to select the user pair $\left(k_{1}[t], k_{2}[t]\right)$ and the transmission strategy $s[t]$ in every time slot $t$ such that the time average rates $\overline{\mathbf{R}}^{*}$ are achieved. In general, it is difficult to find an optimal solution $\overline{\mathbf{R}}^{*}$ for this problem, since the feasible region $\mathcal{R}$ does not admit a simple characterization. However, it has been shown in previous work [11], [12] that a near optimal dynamic scheduling scheme is achievable for problems with a structure similar to that of (11) by applying stochastic network optimization, which will be discussed in the following section.

\section{DynAmic Scheduling Scheme}

In this section, we propose an efficient dynamic scheduling scheme that achieves near optimal performance by applying the stochastic network optimization framework [11], [12]. For a system with infinite queue backlog, the main idea of the aforementioned framework is to design a dynamic scheduling scheme in two steps: The first step is to introduce virtual queues and a virtual arrival process, and design a stable scheduling policy that achieves the stability of all virtual queues whenever the virtual arrival rates are inside the feasible region $\mathcal{R}$. The second step is to find the virtual arrival process with average rates $\overline{\mathbf{A}}$ that approach the optimal point $\overline{\mathbf{R}}^{*}$. By applying the stable scheduling policy to the virtual arrival process, the system will operate at a point $\overline{\mathbf{R}} \succeq \overline{\mathbf{A}}$ (pointwise domination). Since $\varphi(\cdot)$ is non-decreasing, it is guaranteed that the utility achieved is close to the optimal point.

\section{A. Dynamic Scheduling Scheme for Two-cell System}

We apply the stochastic optimization framework to the system considered in Section II. We first introduce the virtual queue $Q_{i k}[t]$ for user $k$ in cell $i$ with virtual arrival rate $A_{i k}[t]$ in slot $t$. The virtual queues evolve according to the following stochastic difference equation

$$
\begin{array}{r}
Q_{i k}[t+1]=\max \left\{Q_{i k}[t]-R_{i k}[t], 0\right\}+A_{i k}[t], \\
\forall k \in \mathcal{K}_{i}, i \in \mathcal{I} .
\end{array}
$$

Then, we propose to use the following stable scheduling policy: in each time slot $t$, select a user pair $\left(k_{1}[t], k_{2}[t]\right)$ 
and corresponding transmission strategy $s[t]$ based on the instantaneous CSI by solving the following problem

$$
\begin{array}{cl}
\underset{k_{1}[t], k_{2}[t], s[t]}{\operatorname{maximize}} & \sum_{i \in \mathcal{I}} Q_{i k_{i}[t]}[t] R_{i k_{i}[t]}[t] \\
\text { subject to } & k_{1}[t] \in \mathcal{K}_{1}, k_{2}[t] \in \mathcal{K}_{2}, \\
& s[t] \in \mathcal{S} .
\end{array}
$$

According to [12], it can be shown that the above scheduling policy guarantees the stability of the system with virtual queues.

Next, we determine the virtual arrival rates in time slot $t$ by solving the following problem

$$
\begin{array}{ll}
\underset{\mathbf{A}_{1}[t], \mathbf{A}_{2}[t]}{\operatorname{maximize}} & \beta \varphi\left(\mathbf{A}_{1}[t], \mathbf{A}_{2}[t]\right)-\sum_{i \in \mathcal{I}} \sum_{k_{i}[t] \in \mathcal{K}_{i}} A_{i k_{i}[t]}[t] Q_{i k_{i}[t]}[t] \\
\text { subject to } & 0 \leq A_{i k_{i}[t]}[t] \leq A_{\max }, \quad \forall k_{i}[t] \in \mathcal{K}_{i}, \quad i \in \mathcal{I},
\end{array}
$$

where $\beta, A_{\max }>0$ are constants, $\mathbf{A}_{i}[t]$ denotes the collection of all virtual arrival rates for users in cell $i$ at time slot $t$.

From the above discussion, we obtain the following dynamic scheduling scheme: in each time slot, we select a pair of users and the corresponding transmission strategy according to (13), and then update the virtual arrival rates and virtual queue length according to (14) and (12), respectively. With this dynamic scheduling scheme, the following results can be obtained.

Proposition 1: For given constants $\beta$ and $A_{\max }$, and a concave and entry-wise non-decreasing utility function $\varphi(\cdot)$, if there exists a point $\overline{\mathbf{R}}^{\dagger} \in \mathcal{R}$ with each element in $\left[0, A_{\max }\right]$, and a constant $\delta \in[0,1]$ such that $\varphi\left(\delta \overline{\mathbf{R}}^{\dagger}\right)>-\infty$, then we have

(a) $\lim _{t \rightarrow \infty} \inf \varphi\left(\frac{1}{t} \sum_{\tau=0}^{t-1} \mathbb{E}[\mathbf{R}[\tau]]\right) \geq \varphi\left(\overline{\mathbf{R}}^{*}\left(A_{\max }\right)\right)-C / \beta$,

where $C>0$ is a constant, $\mathbf{R}[\tau]$ is the collection of the service rates at both base stations in time slot $\tau$, the expectation $\mathbb{E}[\cdot]$ is with respect to the joint probability distribution of channel matrix and scheduling decision under the proposed scheduling scheme, and $\overline{\mathbf{R}}^{*}\left(A_{\max }\right)$ is the solution to problem (11) with the additional constraint $0 \leq \bar{R}_{i k} \leq A_{\max }, \forall k \in \mathcal{K}_{i}, i \in \mathcal{I}$;

$$
\text { (b) } \begin{aligned}
\lim _{t \rightarrow \infty} \sup & \frac{1}{t} \sum_{\tau=0}^{t-1} \sum_{i \in \mathcal{I}} \sum_{k_{i}[\tau] \in \mathcal{K}_{i}} \bar{R}_{i k_{i}[\tau]}^{\dagger} \mathbb{E}\left[Q_{i k_{i}[\tau]}[\tau]\right] \\
& \leq \frac{C+\beta\left[\varphi\left(\overline{\mathbf{R}}^{*}\left(A_{\max }\right)\right)-\varphi\left(\delta \overline{\mathbf{R}}^{\dagger}\right)\right]}{1-\delta} .
\end{aligned}
$$

The proof follows [11], [12], and is omitted for brevity. From $(a)$, when $A_{\max }$ is sufficiently large such that $A_{\max } \geq$ $\bar{R}_{i k}^{*}, \forall k \in \mathcal{K}_{i}, i \in \mathcal{I}$, then (15) implies that the utility achieved by applying the dynamic scheduling scheme can be arbitrarily close to the optimal utility $\varphi\left(\overline{\mathbf{R}}^{*}\right)$ by increasing $\beta$. This comes with the tradeoff that the virtual queue backlog grows linearly with $\beta$, as seen from (16). The virtual queue backlog indicates the delay between the virtual data admitted into the queues and the actual data transmitted, which affects the number of time slots required for the time average rates to become close to their desired values.

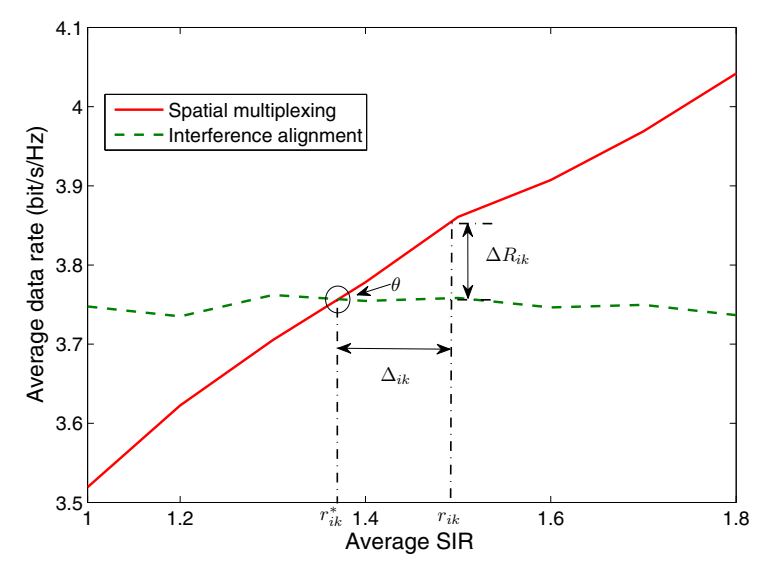

Fig. 2. The average data rate versus the average SIR for both spatial multiplexing and interference alignment strategies with $\mathrm{SNR}=10 \mathrm{~dB}$ and $g_{i k}=0.6$.

\section{B. Efficient Dynamic Scheduling Algorithm}

To implement the proposed dynamic scheduling scheme, in each time slot we need to solve (13), which is a discrete optimization problem that involves implementation of both transmission strategies for a given pair of users (when searching for the optimal solution). For large networks with many users, solving (13) is complex and may not be suitable for real time processing. Therefore, it is desirable to develop an efficient algorithm that can reduce complexity.

A possible way to reduce complexity is to simplify the transmission strategy selection process when solving (13). As discussed in Section II-A, interference alignment is desirable for users who experience significant inter-cell interference, while spatial multiplexing is preferred for others. Fig. 2 shows the average data rate of the two strategies as a function of the average signal-to-interference ratio (SIR) for a two-cell MIMO system with $N_{T}=N_{R}=2$, where the average SIR for user $k$ in cell $i$ is defined as $r_{i k} \triangleq g_{i k} / g_{j k}(i, j \in$ $\mathcal{I}, i \neq j$ ). The result in Fig. 2 is obtained by averaging over $10^{4}$ channel realizations. From Fig. 2, we have the following observations. First, for user $k$ in cell $i(i \in \mathcal{I})$, given the SNR value and the path gain $g_{i k}$, there exists a threshold $r_{i k}^{*}$ such that the average achievable rate of spatial multiplexing is larger than that of interference alignment if the average SIR $r_{i k}$ is greater than $r_{i k}^{*}$. Second, the average achievable rate of interference alignment does not depend on the average interference strength, and remains almost constant for different $r_{i k}$, which can also be verified from (7). Therefore, given the SNR and $g_{i k}$, we can approximate the difference between the average achievable rates of the two strategies as

$$
\Delta R_{i k} \triangleq R_{i k}^{\mathrm{SM}}-R_{i k}^{\mathrm{IA}} \approx \tan \theta \Delta_{i k}
$$

where $\tan \theta>0$ is a constant and $\Delta_{i k} \triangleq r_{i k}-r_{i k}^{*}$ (as shown in Fig. 2).

Based on the above approximation, while solving (13), we introduce an indicator $s_{I}\left(k_{1}[t], k_{2}[t]\right) \triangleq \sum_{i \in \mathcal{I}} \Delta_{i k_{i}[t]} Q_{i k_{i}[t]}[t]$ to facilitate the selection of the transmission strategy $s[t]$ for 
a given user pair $\left(k_{1}[t], k_{2}[t]\right)$, where we set

$$
s[t]= \begin{cases}1, & \text { if } s_{I}\left(k_{1}[t], k_{2}[t]\right) \geq 0, \\ 0, & \text { otherwise. }\end{cases}
$$

The rational behind (18) is as follows. From (17), we have

$$
s_{I}\left(k_{1}[t], k_{2}[t]\right) \approx \frac{1}{\tan \theta} \sum_{i \in \mathcal{I}} Q_{i k_{i}[t]}[t]\left(R_{i k_{i}[t]}^{\mathrm{SM}}-R_{i k_{i}[t]}^{\mathrm{IA}}\right)
$$

where $R_{i k_{i}[t]}^{\mathrm{SM}}$ and $R_{i k_{i}[t]}^{\mathrm{IA}}$ represent the average achievable data rate using spatial multiplexing and interference alignment, respectively. Eq. (19) implies that when $s_{I}\left(k_{1}[t], k_{2}[t]\right)>0$, spatial multiplexing achieves a better performance on average, while interference alignment outperforms spatial multiplexing on average when $s_{I}\left(k_{1}[t], k_{2}[t]\right)<0$. For each user $k$ ( $k \in \mathcal{K}_{i}, i \in \mathcal{I}$ ), the average $\operatorname{SIR} r_{i k}$ and the corresponding threshold value $r_{i k}^{*}$ can be calculated offline before the scheduling begins. Then, in time slot $t$, for any given user pair $\left(k_{1}[t], k_{2}[t]\right), s_{I}\left(k_{1}[t], k_{2}[t]\right)$ can be obtained immediately. Therefore, by using (18) to determine the transmission strategy when searching the solution to (13), the complexity is reduced since we do not need to consider both strategies for a particular pair of users.

By using the above transmission strategy selection method, we propose an efficient dynamic scheduling algorithm (DSA) as shown in Algorithm 1.

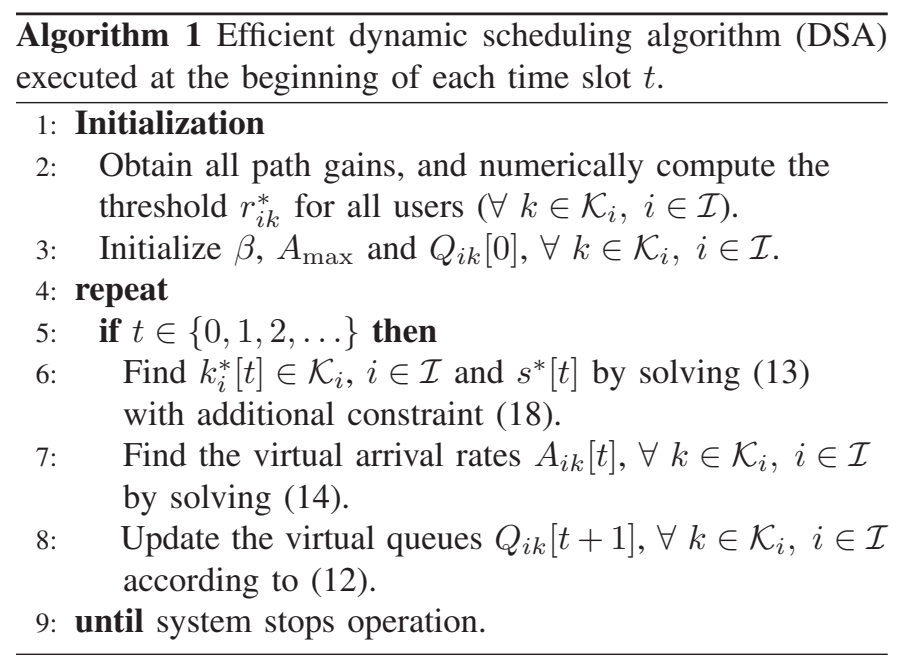

In Algorithm 1, the scheduler first obtains the path gains of all links from the two base stations and calculate the SIR thresholds for all users. Meanwhile, it initializes the scheduling parameters and all the virtual queues. When scheduling starts, the scheduler follows Step 6 to select users and transmission strategy, and then updates the virtual arrival rates and virtual queues in Steps 7 and 8, respectively. Note that in Step 6 , we impose constraint (18) when solving (13) to reduce complexity. However, the decision rule of (18) is based on the average performance of the two transmission strategies, and also involves an approximation. Hence, the above algorithm may not find the optimal solution to (13) for each time slot.

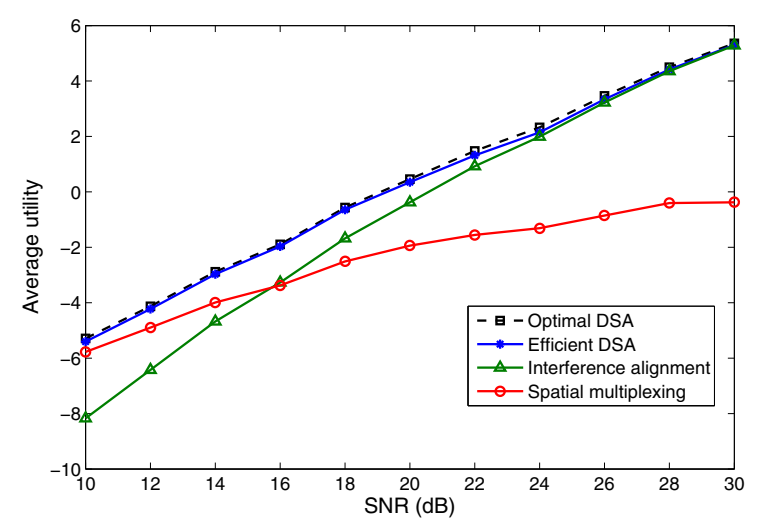

Fig. 3. Average utility versus the SNR.

\section{Performance Evaluation}

In this section, we evaluate the performance of the proposed dynamic scheduling scheme and compare it with scheduling schemes where a single transmission strategy is employed. Consider a two-cell network where the radius of each cell is $50 \mathrm{~m}$ and two base stations are located at the cell centers with distance $d$. In each cell, 10 users are randomly deployed, with the restriction that at least $N_{e}$ of them are cell-edge users. A cell-edge user in cell $i(i \in \mathcal{I})$ is located at a position where the distance to base station $i$ is between $45 \mathrm{~m}$ and 50 $\mathrm{m}$, and the angle between the direction from base station $i$ to the user and the direction from base station $i$ to base station $j(j \neq i)$ is less than $30^{\circ}$. Each node in the system (including both base stations and all users) is equipped with two omnidirectional antennas $\left(N_{R}=N_{T}=2\right)$. The wireless channels undergo path loss with path loss exponent 2 and Rayleigh fading. Unless specified, the above settings are valid for all figures in this section.

The spatial multiplexing and interference alignment transmission strategies are implemented according to Section II. For scheduling, we adopt the utility function $\varphi\left(\bar{R}_{1}, \bar{R}_{2}\right)=$ $\sum_{i \in \mathcal{I}} \sum_{k \in \mathcal{K}_{i}} \log \left(\bar{R}_{i k}\right)$ which achieves proportional fairness. With this utility function, we obtain the arrival rate for the virtual queues by solving (14), which leads to $A_{i k_{i}[t]}[t]=$ $\min \left\{\beta / Q_{i k_{i}[t]}[t], A_{\max }\right\}$. The initial length of all virtual queues is set to $1 \mathrm{bit} / \mathrm{Hz}$. Other parameters are $A_{\max }=$ $100, \beta=100$. We simulate the proposed efficient DSA for different SNR values, different distances between the two base stations $(d)$, and different number of cell-edge users $\left(N_{e}\right)$, where the SNR represents the average received SNR at a reference distance of $50 \mathrm{~m}$. We also implement two scheduling schemes with a single transmission strategy, spatial multiplexing and interference alignment, respectively, using the stochastic network optimization framework.

The utility for different SNRs with $d=100 \mathrm{~m}$ and $N_{e}=3$ is shown in Fig. 3. It is shown that the proposed efficient DSA outperforms the schemes with a single transmission strategy. Specifically, as the SNR decreases, the proposed DSA approaches the scheme with spatial multiplexing, since in the low SNR region, both the signal and interference power are small and noise dominates the performance for 


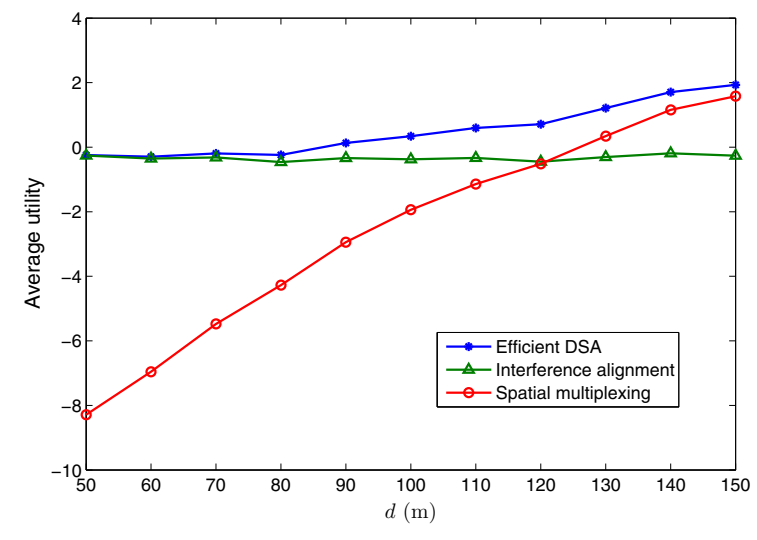

Fig. 4. The average utility versus the distance $d$.

almost all the users. As the SNR increases, the noise power becomes insignificant and eliminating interference becomes critical. Hence, interference alignment outperforms spatial multiplexing for almost all the users, and the proposed DSA approaches the scheduling scheme with interference alignment. We observe a performance improvement by employing the proposed DSA when the SNR is between $12 \mathrm{~dB}$ and $22 \mathrm{~dB}$, which implies that in this SNR region, inter-cell interference dominates the performance at some (but not all) users, and a proper transmission strategy should be selected for each user to achieve the optimal performance. It is also shown that the proposed efficient DSA achieves a performance close to that of the optimal DSA (which solves (13) optimally).

Fig. 4 shows the utility as a function of the distance between the base stations, with an SNR of $20 \mathrm{~dB}$ and $N_{e}=3$. It can be seen that when $d$ decreases, the proposed DSA approaches the scheme with interference alignment. The reason is that the inter-cell interference increases as $d$ decreases, and dominates the performance, especially for cell-edge users. The proposed DSA approaches the scheme with spatial multiplexing as $d$ increases, since the inter-cell interference becomes insignificant when the two base stations are far from each other.

Fig. 5 shows the utility with respect to different numbers of cell-edge users $\left(N_{e}\right)$ with $\mathrm{SNR}=10 \mathrm{~dB}$ and $d=100 \mathrm{~m}$. The performance of efficient DSA approaches that of interference alignment as $N_{e}$ increases, since on average interference alignment achieves a higher data rate than spatial multiplexing for users in the cell-edge region. When there are few cell-edge users, spatial multiplexing achieves higher data rate for most of the users, and the performance of the efficient DSA is close to that of the scheme with spatial multiplexing.

\section{CONCLUSION}

In this paper, we proposed a dynamic downlink scheduling scheme for two-cell MIMO networks. We employed interference alignment to improve the data rate of cell-edge users whose performance is limited by inter-cell interference. We applied the stochastic network optimization framework and designed an efficient dynamic scheduling scheme which jointly selects users and a corresponding transmission strategy in each time slot. Numerical results showed that our proposed scheme

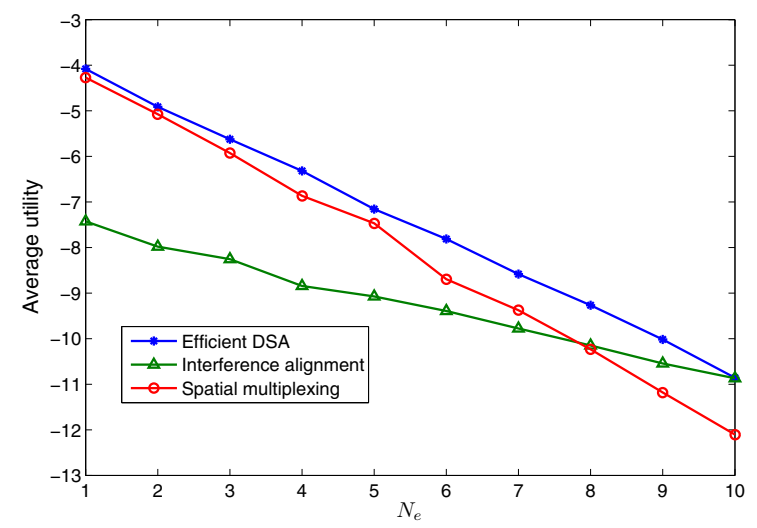

Fig. 5. The average utility versus the number of cell-edge users $N_{e}$.

outperforms schemes with a single transmission strategy especially when inter-cell interference dominates the performance of some users. An interesting topic for future work is the extension of the proposed dynamic scheduling scheme to multi-user multi-cell scenarios, and the development of a distributed scheduling algorithm with imperfect CSI.

\section{ACKNOWLEDGMENT}

This research is supported by the Natural Sciences and Engineering Research Council (NSERC) of Canada and the Institute for Computing, Information and Cognitive Systems (ICICS) at the University of British Columbia.

\section{REFERENCES}

[1] S. Parkvall, A. Furuskar, and E. Dahlman, "Evolution of LTE toward IMT-Advanced," IEEE Comm. Mag., vol. 49, pp. 84-91, Feb. 2011.

[2] D. Tse and P. Viswanath, Fundamentals of Wireless Communication. Cambridge University Press, 2005.

[3] D. Gesbert, S. Hanly, H. Huang, S. S. Shitz, O. Simeone, and W. Yu, "Multi-cell MIMO cooperative networks: A new look at interference," IEEE J. Sel. Areas Commun., vol. 28, pp. 1380-1408, Dec. 2010.

[4] V. R. Cadambe and S. A. Jafar, "Interference alignment and degrees of freedom of the K-user interference channel," IEEE Trans. on Information Theory, vol. 54, pp. 3425-3441, Aug. 2008.

[5] R. Tresch and M. Guillaud, "Clustered interference alignment in large cellular networks," in Proc. of IEEE PIMRC, Tokyo, Japan, Sept. 2009.

[6] C. Suh, M. Ho, and D. Tse, "Downlink interference alignment," in Proc. of IEEE GLOBECOM, Miami, FL, Dec. 2010.

[7] W. Shin, N. Lee, J. Lim, C. Shin, and K. Jang, "On the design of interference alignment scheme for two-cell MIMO interfering broadcast channels," IEEE Trans. on Wireless Commun., vol. 10, pp. 437-442, Feb. 2011.

[8] F. Kelly, A. Maulloo, and D. Tan, "Rate control in communication networks: Shadow prices, proportional fairness and stability," Journal of the Operational Research Society, vol. 49, pp. 237-252, 1998.

[9] A. Sang, N. Lee, X. Wang, M. Madihian, and R. D. Gitlin, "A flexible downlink scheduling scheme in cellular packet data systems," IEEE Trans. on Wireless Commun., vol. 5, pp. 568-577, Mar. 2006.

[10] H. Shirani-Mehr, H. Papadopoulos, S. A. Ramprashad, and G. Caire, "Joint scheduling and Hybrid-ARQ for MU-MIMO downlink in the presence of inter-cell interference," in Proc. of IEEE ICC, Cape Town, South Africa, May 2010.

[11] H. Shirani-Mehr, G. Caire, and M. J. Neely, "MIMO downlink scheduling with non-perfect channel state knowledge," IEEE Trans. on Commun., vol. 58, pp. 2055-2066, July 2010.

[12] L. Georgiadis, M. J. Neely, and L. Tassiulas, "Resource allocation and cross-layer control in wireless networks," Foundations Trends Networking, vol. 1, pp. 1-149, 2006. 